\title{
Chemical History of Algol and its Components
}

\author{
V. Kolbas ${ }^{1}$, K. Pavlovski ${ }^{1,2}$, J. Southworth ${ }^{2}$, C.-U. Lee ${ }^{3}$, J. W. Lee ${ }^{3}$, \\ S.-L. Kim ${ }^{3}$ and H.-I. Kim ${ }^{3}$ \\ ${ }^{1}$ Department of Physics, Facult of Science, University of Zagreb, Croatia \\ ${ }^{2}$ Astrophysics Group, Keele University, Staffordshire, ST5 5BG, UK \\ ${ }^{3}$ Korea Astronomy and Space Science Institute, Yuseong-yu, Daejeon, Korea
}

\begin{abstract}
We present a new observational project to study the hierarchical triple stellar system Algol, concentrating on the semidetached eclipsing binary at the heart of the system. Over 140 high-resolution and high-S/N spectra have been secured, of which 80 are from FIES at the Nordic Optical Telescope, La Palma, and the remainder were obtained with BOES at the Bohyunsan Optical Astronomy Observatory in Korea. All three components were successfully detected by the method of spectral disentangling, which yields the individual spectra of the three stars and also high-quality spectroscopic elements for both the inner and outer orbits. We present a detailed abundance study for the mass-accreting component in the inner orbit, which holds information on the history of mass transfer in the close inner binary system. We also reveal the atmospheric parameters and chemical composition of the tertiary component in the outer orbit.
\end{abstract}

Keywords. stars: binaries: eclipsing — stars: fundamental parameters - stars: chemically peculiar — stars: binaries: spectroscopic — stars: early-type

\section{New spectroscopy of Algol}

Algol ( $\beta$ Persei, HD 19356) is on of the most important stellar systems for studying mass transfer and mass loss in an interacting binary because it contains the brightest eclipsing binary with deep eclipses. It is the prototype of the class of close binaries in which mass reversals occurred due to rapid mass transfer, resulting in ongoing mass transfer from the G-K sub-giant secondaries to the B5-A5 main-sequence primaries. In spite of a rich observational history, Algol is still lacking definitive understanding although progress has been made in the last decade (see Retter, Richards \& Wu 2005). Its brightness is favourable for new observational techniques, recently allowing Zavala et al. (2011) to fully spatially resolve its components. However, Algol has persistently lacked extensive high-precision photometric and spectroscopic observations.

Therefore, in 2006 we started a new Échelle spectroscopic programme to observe Algol (Pavlovski et al. 2010). Over 80 high-resolution and high-S/N Échelle spectra were secured in two observing runs with FIES at the Nordic Optical Telescope on La Palma, Spain. The spectral coverage of these observations is $3640-7380 \AA$, and the resolving power is $R=47000$. Over 60 additional Échelle spectra were secured since 2009, at the Bohyunsan Optical Astronomy Observatory in Korea. Two different fibres of the Bohyunsan Optical Echell Spectrography (BOES) have been used, giving $R=45000$ and $R=30000$. The spectral range is $3500-10000 \AA$ and the typical $\mathrm{S} / \mathrm{N}$ is 400 . 


\section{Chemical composition of the components of the Algol system}

Spectral disentangling has been performed on these data as described in Pavlovski et al. (2010). Algol is a hierarchical triple system with a third component in a wide orbit of period $640 \mathrm{~d}$. The contribution of the third component to the total light of the system is uncertain (Richards et al. 1988), but is significantly larger than that of the sub-giant component in the inner semidetached binary. The best ground-based photometry (Kim 1989) is not of sufficient quality to give the precise light contributions of the three stars, so we must seek these light dilution factors from spectroscopy.

Previous estimates of the spectral type of the third component cover a broad range, from a mid-F to a late A-type star with metallic characteristics. Therefore, we calculated a grid of synthetic spectra covering $T_{\text {eff }}=7000-8600 \mathrm{~K}$ in steps of $200 \mathrm{~K}$. We fixed $\log g=$ 4.3. By varying the light dilution factor, we calculated the sum of squared residuals between theoretical and disentangled (separated) spectra. The best match is found for $T_{\text {eff }}=7600 \mathrm{~K}$, and light dilution factor 0.07 . The depth of some spectral lines is almost independent of $T_{\text {eff }}$, making them sensitive indicators of the light dilution factor. We corroborate the weakness of the CaII lines in the spectrum of the third component, and a slight underabundance of Sc, another classical indicator of a metallic-lined stars. The third component may be mildly metallic in nature, and a detailed spectroscopic abundance analysis will be able to confirm or refute this.

Armed with an improved measurement of the contribution of the third component to the total luminosity of the system, we turned back to the $B$ and $V$ light curves from Kim (1989). We now find that the primary is contributing $90.5 \%$ of the total system's light outside eclipse. The renormalized disentangled spectrum of the primary will next be used to estimate its $T_{\text {eff }}$ (its $\log g$ is known from the dynamics of the inner system), and derive the chemical composition of its photosphere.

From fitting the Balmer lines and then fine-tuning using Fe II lines, we find for the primary star $T_{\text {eff }}=12950 \mathrm{~K}$. Profiles of several prominent He I lines have been calculated in NLTE, and indicate a solar helium abundance for this star. NLTE calculations for the CNO elements indicate a slight deficiency of $\mathrm{C}$ relative to $\mathrm{N}$, in line with the results of Cugier \& Hardrop (1988) and Tomkin et al. (1993). Abundances for other elements have been calculated in LTE, and indicate a slight underabundance of -0.1 dex relative to solar. A more detailed examination is needed before definitive conclusions could be drawn on this issue.

\section{Acknowledgements}

KP acknowledges receipt of a Leverhulme Visiting Professorship which enabled him to stay at Keele University where a part of this work was performed.

\section{References}

Cugier, H. \& Hardrop, I. 1988, A\& A, 202, 101

Kim, H.-I. 1989, ApJ, 342, 1061

Pavlovski, K., Kolbas, V., \& Southworth, J. 2010, ASP-CS, 435, 247

Retter, A., Richards, M. T., \& Wu, K. 2005, ApJ, 621, 417

Richards, M. T., Mochnacki, S. W., \& Bolton, C. T. 1988, AJ, 96, 326

Tomkin, J., Lambert, D. L., \& Lemke, M. 1993, MNRAS, 265, 581

Zavala, R. T., Hummel, C. A., Boboltz, D. A., Ojha, R., Shaffer, D. B., Tycner, C., Richards, M. T., \& Hutter, D. J. 2011, ApJL, 715, L44 